Document downloaded from:

http://hdl.handle.net/10251/79235

This paper must be cited as:

Bockelt, AS.; Vidal Rodriguez, B. (2017). Dynamic Tuning of Mean Carrier Momentum Relaxation Time in InGaAs InAIAs THz Photoconductive Switches. IEEE Transactions on Terahertz Science \&amp; Technology. 7(1):107-113. doi:10.1109/TTHZ.2016.2636742.

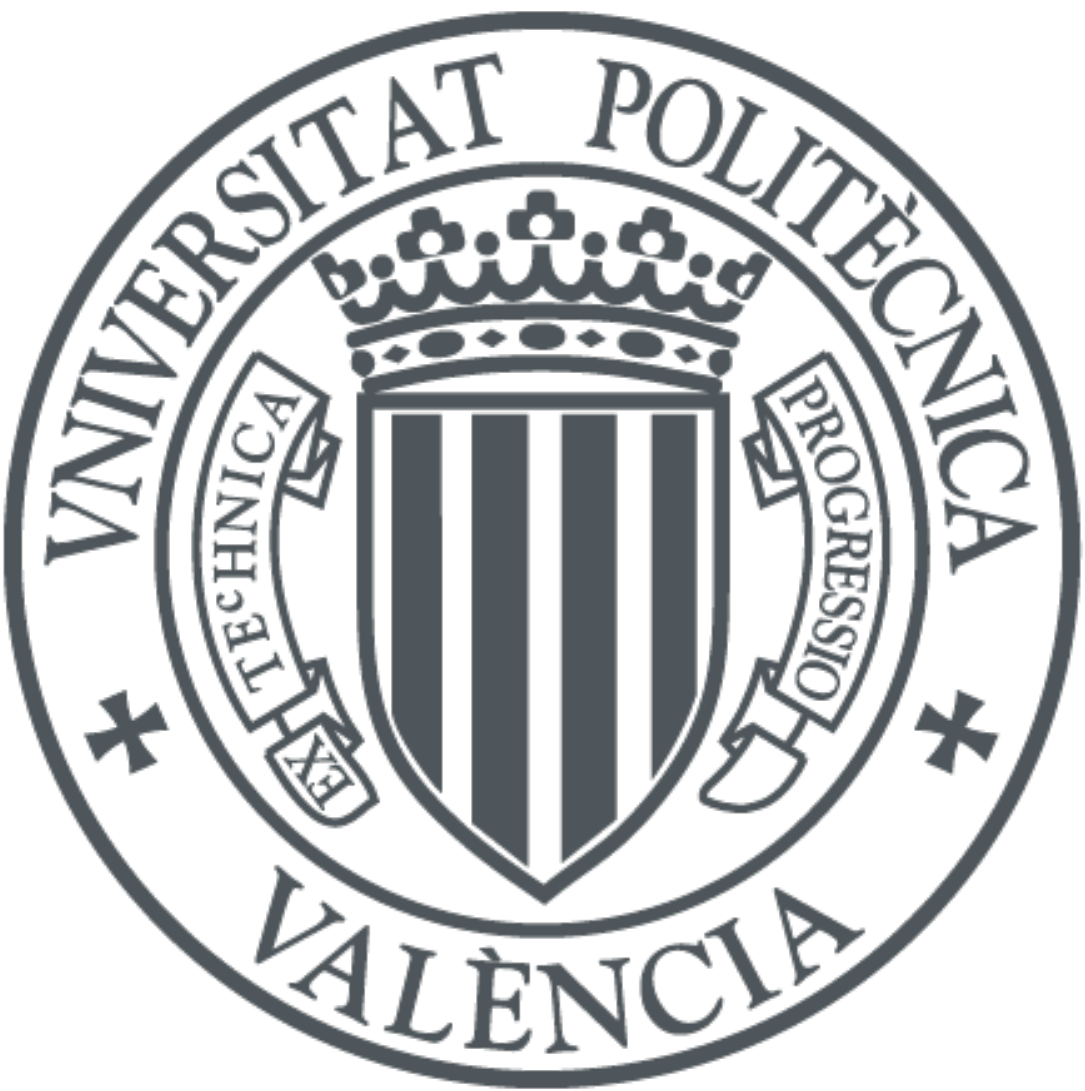

The final publication is available at

http://dx.doi.org/10.1109/TTHZ.2016.2636742

Copyright Institute of Electrical and Electronics Engineers (IEEE)

Additional Information 


\title{
Dynamic tuning of mean carrier momentum relaxation time in InGaAs/InAlAs THz photoconductive switches
}

\author{
Alexander Bockelt and Borja Vidal, Senior Member, IEEE
}

\begin{abstract}
The carrier transport in InGaAs/InAlAs multilayer hetero-structures used for the implementation of $\mathrm{THz}$ photoconductive antennas is investigated under the injection of a continuous optical wave. By varying the amplitude level of the continuous optical wave in the transmitter or receiver antennas used in a conventional pulsed THz time-domain spectrometer, the amplitude of the detected photocurrent can be controlled without affecting its bandwidth. Unlike increasing the optical power of the pulsed signal, it is shown that raising the continuous optical power results in a reduction of the measured photocurrent. This lowering of the conductivity can be explained by changes in the instantaneous carrier momentum relaxation time in the material rather than variations of the free carrier density level. This behavior affects in systems showing optical continuous-wave components, as for example, fiber-based $\mathrm{THz}$ time domain spectrometers including optical amplifiers.
\end{abstract}

\section{INTRODUCTION}

The control of material properties such as electronic energy band gaps, free carrier density and carrier mobility has allowed the development of high performance electronic and optoelectronic devices operating in a wide range of frequency bands. One of the last accessible spectral regions has been the Terahertz (THz) band, where the optical excitation of biased semiconductor photoconductive switches by ultra-short laser pulses has become a widespread method for $\mathrm{THz}$ generation and detection. This progress has made Terahertz time-domain spectroscopy (THz-TDS) a new tool for sensing in industrial and medical applications, such as real-time monitoring in industrial quality control, security scanning, $\mathrm{THz}$ imaging and biomedical sensing [1-3]. To enhance the performance in terms of output power, conversion efficiency and, particularly, bandwidth, an ongoing search for enhanced semiconductor compositions and structures is taking place. Whereas in the infancy of $\mathrm{THz}$ technology radiationdamaged silicon-on-sapphire (RD-SOS) and later low temperature-grown (LTG) GaAs had been the materials of choice, lately photo switches consisting of more sophisticated semiconductor multilayer hetero structures (MLHSs) have been designed and fabricated, which boosted their performance remarkably [4]. An ongoing optimization of device parameters is based on the use of novel semiconductor compounds with electronic bandgaps that can be bridged by optical excitation at $1550 \mathrm{~nm}$, which permit the use of lowcost, compact and mature telecommunications light sources. Additionally, research focuses on the deployment of fiberbased, rather than free space components for optical pulse distribution, which are more robust and easier to implement in industrial scenarios [4-6]. In state-of-the-art devices, common materials are Beryllium-doped low temperaturegrown InGaAs with InAlAs spacer-layers $[4,5]$ and, ultimately, un-doped InGaAs/InAlAs-MLHS grown at standard InGaAs epitaxy growth-temperatures [7, 8]. Further optimization comprises micro structuring, such as the introduction of mesa-ridges for enhanced field distribution [9] and miniaturized electrode gaps for ballistic carrier transport [10]. Most of these enhancements target the key parameters of photoconductors, which principally are the dark resistivity and carrier mobility [11]. These, in turn, depend on time constants and effective masses of the involved charge carriers. In photoconductors, excited electrons and holes contribute to the electronic charge transport only during their lifetime, i.e. while occupying conduction band states and before either recombination or trapping by crystal defects. The progressive development of ultra-fast photoconductors has reduced carrier lifetimes from dozens of picoseconds to the sub-picosecond time-scale and thus has enabled the generation of ultra-short current transients, whose respective spectral contents were vastly extended. Besides free carrier lifetime, studies [12] also include the momentum relaxation time of the carriers, a characteristic mean time between scattering events, which can be associated to the heat of the crystal lattice. Both time constants are determined by the number of potential scattering targets or recombination partners in the material. Implantation of crystal impurities represents a static method of technological control of those parameters, whereas the density of free charge carriers and phonons can be dynamically controlled via the incident optical flux.

In this paper, the effect of a continuous optical wave (CW) on the carrier dynamics and performance of both a $\mathrm{THz}$ photoconductive emitter as well as a receiver is studied, when this CW signal is superposed to the train of femtosecond pulses that the modules are conventionally fed with. Experimental observations are discussed and a microscopic 
model of the carrier dynamics, explaining the system behavior, is presented.

\section{EXPERIMENTAL SETUP}

Experiments were carried out with a fiber-based THzTDS setup (Fig.1) that was implemented with commercial components. The key components are the $\mathrm{THz}$ emitter photoconductive antenna ( $\mathrm{T}_{\mathrm{x}}$ PCA) made up of an InGaAs/InAlAs MLHSs grown at standard InGaAs epitaxy temperatures [8] and a receiver $\left(\mathrm{R}_{\mathrm{x}}-\mathrm{PCA}\right)$ made up of Bedoped LTG-InGaAs/InAlAs MLHSs [4]. The emitter chip consisted of a strip line-type antenna structure of gold interrupted by a laterally contacted semiconductor ridge. The semiconductor compound is a molecular beam epitaxial (MBE)-grown MLHS with alternating InGaAs (thickness: 12 $\mathrm{nm}$ ) and InAlAs spacer layers (thickness: $8 \mathrm{~nm}$ ) on an InPsubstrate. The electrode gap represented by this ridge measures $50 \mu \mathrm{m}$ and hence applicable bias voltages lead to electric field strengths of few $\mathrm{kV} / \mathrm{cm}$. In contrast, the receiver chip had a dipole-structured antenna with a smaller gap of only $10 \mu \mathrm{m}$. The respective MLHSs assure carrier lifetimes of both devices well below 1 ps.

A mode-locked fiber laser centered at $1560 \mathrm{~nm}$ served as optical source. Both PCAs were fed by a train of optical pulses emitted by this ultra-fast laser with a pulse repetition rate of $100 \mathrm{MHz}$ and a pulse duration of around $100 \mathrm{fs}$ at full width at half maximum (FWHM). A thoroughly tailored fiber patch cord of few meters of length, which ensured compensation for chromatic dispersion and a temporal match of the $\mathrm{THz}$ signal and the gate signal at the receiver site, was designed for the optical signal distribution. Temporal traces of the THz-signal were sampled using an optical delay stage, which was implemented in the receiver pathway. To filter out signal-obscuring noise, lock-in-detection with preceding trans-impedance amplification was performed. The signal was modulated for lock-in detection, applying the output voltage of a signal generator at a few $\mathrm{kHz}$ as bias to the emitter PCA and a synchronized reference signal to the lockin amplifier (LIA). A bipolar rectangular waveform was chosen to bias the antenna alternately with the maximum attainable voltage level of $10 \mathrm{~V}$. To inject the $\mathrm{CW}$ signals, fiber couplers with a coupling ratio of 50:50 were inserted in the fiber pathway. A laser source at $1560 \mathrm{~nm}$ was employed as the CW signal source. A further fiber splitter with splitting ratio 99:1 was integrated for power monitoring.

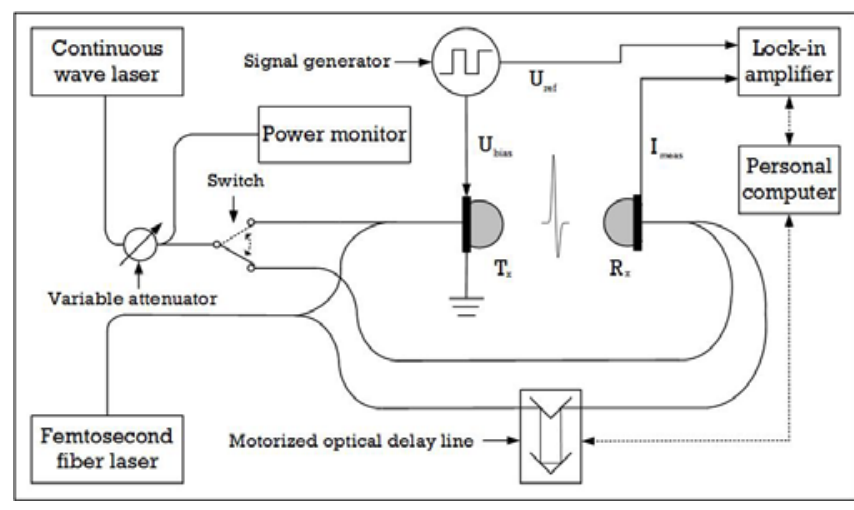

FIG. 1 Experimental setup: The ultrafast fiber laser delivers gate pulses to both the emitter $\left(T_{x}\right)$ and receiver $\left(R_{x}\right)$ PCAs as in standard operation conditions. Additionally, the $\mathrm{CW}$ is either injected into the emitter or receiver pathway.

\section{RESULTS}

The CW signal from the laser source was injected into either the emitter or the receiver PCA. Standard pulsed-only operation was taken for reference. In Fig. 2 the captured timedomain data of these three configurations (CW injection into the emitter, receiver or no $\mathrm{CW}$ injection) are presented. It can be seen that $\mathrm{CW}$ injection in either PCA reduces the photocurrent considerably. For a fixed optical CW power, the amplitude reduction is larger, when the CW is injected into the receiver. Fig. 3 shows a set of time traces captured for successively increasing CW optical powers injected into the receiver PCA. Raising the power from zero to the level of the pulsed signal (few tens of $\mathrm{mW}$ ), the detected current amplitudes are continuously reduced. Normalization of the time traces to their peak amplitudes shows that the waveform is preserved (inset of Fig. 3). Fig. 4 shows spectra corresponding to current transients captured with absent, intermediate and high (with respect to the pulsed optical power) CW powers at the receiver PCA. Within the limit of the available resolution $(\Delta \mathrm{f}=20 \mathrm{GHz})$ and apart from a slight degradation of the dynamic range (DR) due to reduced amplitudes and additional noise, the corresponding spectral data show no significant differences, i.e. no spectral redistribution of power is observed. The peak amplitudes of the photocurrent with respect to the ratio of $\mathrm{CW}$ to pulsed power are presented in Figure 5. The amplitude decreases with rising $\mathrm{CW}$ power, following a reciprocal curve. At equal power levels $\left(P_{C W} / P_{P u l s} \cong 1: 1\right)$ this reduction, if the injection is applied to the receiver, is about $35 \%$. If the $\mathrm{CW}$ injection is applied to the emitter, the amplitude reduction is noticeably smaller (around 8\%). Replacing the CW signal by an ASE noise signal of equivalent power yields similar results (inset of Fig. 5). 


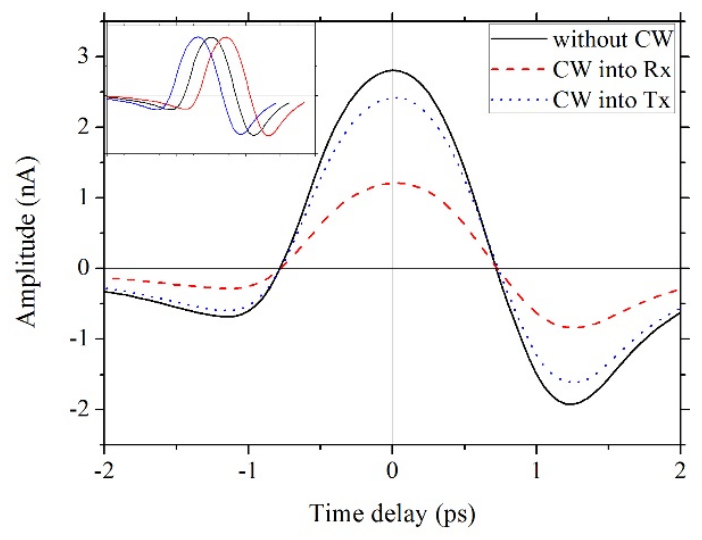

FIG. 2 Detector photocurrents when the $\mathrm{THz}$ time-domain spectrometer is operated conventionally (black solid line), and when additional optical CWs are injected into either the emitter (blue dotted line) or the receiver (red dashed line). Inset: Normalized waveforms, taken in approx. 4 min.

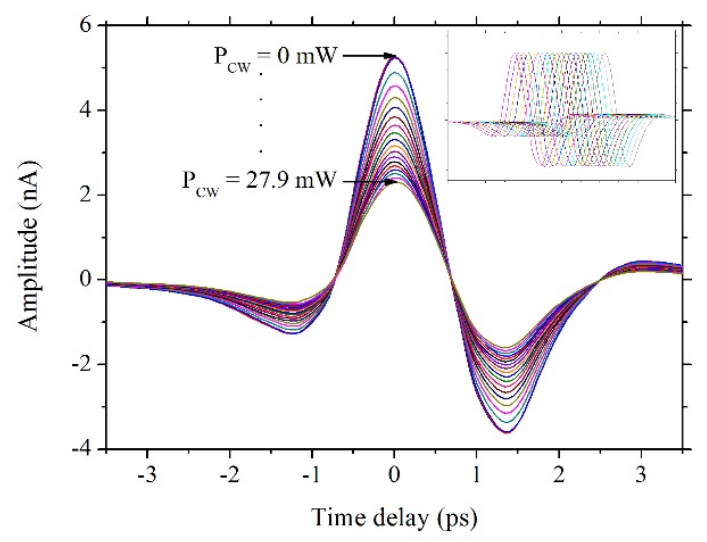

FIG. 3 Time traces captured under variation of additionally injected CW optical power into the receiver PCA. The power was successively increased from $0 \mathrm{~mW}$ to $27.9 \mathrm{~mW}$. Inset: Normalized waveforms.

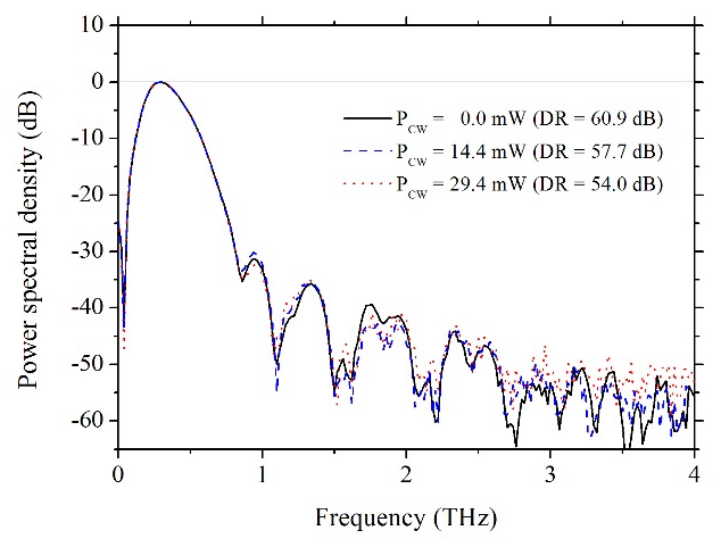

FIG. 4 Fourier-spectra of detector currents with absent, intermediate and high CW optical powers injected into the receiver PCA. Dips in the spectra are caused by residual polarization mode dispersion (PMD) in the fiber patch cords. PMD generates delayed versions of the optical pulses, what leads to filtering at equidistant frequencies.

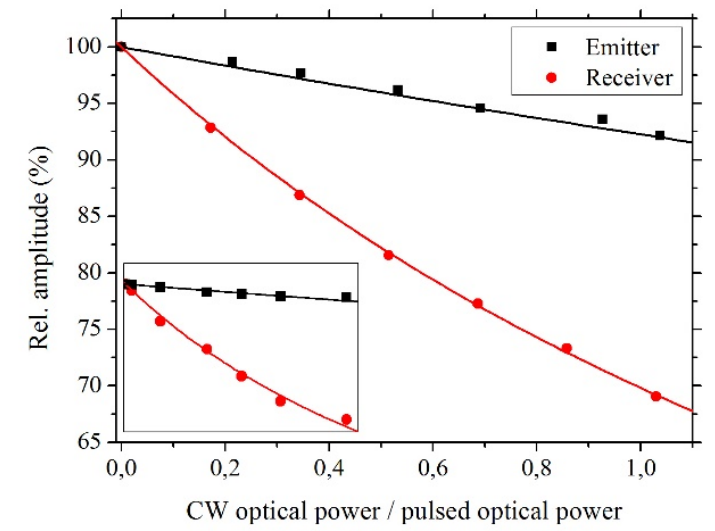

FIG. 5 Detector current amplitude vs. continuous to pulsed wave power ratio. Data are fitted with a reciprocal function. Inset: Replacing the CW power by an equivalent ASE optical amplifier noise power.

\section{DISCUSSION}

It has been shown that for Be-doped LTG-InGaAs/InAlAs photoconductors, the principal mechanism governing their carrier dynamics is the electron capture by ionized $\mathrm{As}_{\mathrm{Ga}}{ }^{+}$antisites acting as trapping centers [13]. The fast trapping process, taking place in the sub-ps range, has turned out to dominate over electron-hole recombination, which has been found to be of the order of tens to hundreds of ps [8]. The growth temperature defines the density of $\mathrm{As}_{\mathrm{Ga}}$ anti-sites. The 
Be-dopant implantation proves responsible for their ionization to build up the $\mathrm{As}_{\mathrm{Ga}}{ }^{+}$electron-traps and hence the defect level associated to this fast trapping process. Moreover, Be-dopants form hole traps in the valence-band, which in turn accelerate the recombination rate [13]. Analogously, in un-doped InGaAs/InAlAs grown at standard epitaxy-growth-temperatures, InAs- or AlAs-like alloy clusters in the InAlAs-layers form trapping centers [7]. Low defect levels or high optical excitation fluxes can lead to the filling of a considerable part of trapping centers and variations of the number of scattering targets. Thus, the carrier lifetimes in the conduction band as well as carrier scattering rates are subject to change. Whereas the defect level represents a built-in property of the photo-switch, a variable optical flux dynamically affects the time constants.

In conventional THz-TDS in the same range of optical powers, an increase of the pulsed optical flux has shown to raise the detected photocurrents. However, the injection of the $\mathrm{CW}$ in the experiments led to a considerable reduction of the measured detector signal. Measurements of the average DC conductivity of the PCAs, when exposed to the CW, instead, show an increase with rising optical CW power, as it could be expected. Several microscopic processes have been considered as the possible cause of the observed effect.

Firstly, considerations are made on how the CW affects the generation process. Since the radiated $\mathrm{THz}$ field strength $E_{T H z}(t)$ scales with the temporal variation of the photoinduced current density $d / d t(j(t))$ in the emitter PCA [12] and the latter with the free carrier density $n(t)$ as well as with the conduction band carrier velocity $v(t), E_{T H z}(t)$ is proportional to the carrier accumulation rate in the conduction band $d / d t(n(t))$ and the corresponding carrier acceleration $d / d t v(t))$ [14]:

$$
\begin{aligned}
E_{T H Z}(t) \propto \frac{d}{d t} j(t) & =\frac{d}{d t}(e n(t) v(t)) \\
& =e\left(v(t) \frac{d}{d t} n(t)\right. \\
& \left.+n(t) \frac{d}{d t} v(t)\right)
\end{aligned}
$$

The time-dependence of the quantities $n(t)$ and $v(t)$ is given implicitly via the incident optical power, so the equation becomes:

$$
\begin{aligned}
& E_{T H z}\left(P_{o p t}(t)\right) \\
& \propto\left(v\left(P_{o p t}(t)\right) \frac{d}{d t} n\left(P_{o p t}(t)\right)\right. \\
& \left.+n\left(P_{o p t}(t)\right) \frac{d}{d t} v\left(P_{o p t}(t)\right)\right) \\
& =\left(v\left(P_{o p t}(t)\right) \frac{\partial n\left(P_{o p t}\right)}{\partial P_{o p t}} \frac{d P_{o p t}(t)}{d t}\right. \\
& \left.+n\left(P_{o p t}(t)\right) \frac{\partial v\left(P_{o p t}\right)}{\partial P_{o p t}} \frac{d P_{o p t}}{d t}\right) .
\end{aligned}
$$

In the following, a prime denotes quantities after taking into account the added CW. An additional CW adds the optical power $P_{C W}$ to the pulsed optical power $P_{p u l s}(t)$ to make up the total incident optical power $P_{o p t}^{\prime}(t)$. On the time scale of the pulse duration, much longer than the CW's optical cycles, $P_{C W}$ is considered constant. Thus, quasi-static CW power leaves the temporal derivatives of the pulses unchanged:

$$
\begin{gathered}
\frac{d P_{o p t}^{\prime}(t)}{d t}=\frac{d P_{p u l s}(t)}{d t}+\underbrace{\frac{d P_{C W}}{d t}}_{=0}=\frac{d P_{p u l s}(t)}{d t} \\
=\frac{d P_{o p t}(t)}{d t} .
\end{gathered}
$$

With Eq. 3 the expression for $E_{T H z}^{\prime}$ becomes:

$$
\begin{gathered}
E_{T H z}^{\prime}\left(P_{o p t}^{\prime}(t)\right) \\
\propto\left(v^{\prime}\left(P_{o p t}^{\prime}(t)\right) \frac{\partial n^{\prime}\left(P_{o p t}\right)}{\partial P_{o p t}} \frac{d P_{o p t}(t)}{d t}\right. \\
\left.+n^{\prime}\left(P_{o p t}^{\prime}(t)\right) \frac{\partial v^{\prime}\left(P_{o p t}\right)}{\partial P_{o p t}} \frac{d P_{o p t}}{d t}\right) .
\end{gathered}
$$

The carrier population $n^{\prime}\left(P_{o p t}^{\prime}(t)\right)$ has two contributions $n\left(P_{\text {puls }}(t)\right)$ and $n\left(P_{C W}(t)\right)$ with the latter term always being an increment. Hence, it holds $n^{\prime}\left(P_{o p t}^{\prime}(t)\right)>n\left(P_{o p t}(t)\right)$. In the non-saturation regime $n\left(P_{o p t}(t)\right)$ is assumed linearly growing and the respective partial derivative $\partial n^{\prime}\left(P_{o p t}^{\prime}\right) /$ $\partial P_{o p t}^{\prime}$ equal to $\partial n\left(P_{\text {opt }}\right) / \partial P_{\text {opt }}$. Comparing the terms of Eq. 2 and Eq. 4 one infers that $E_{T H z}^{\prime}(t)<E_{T H z}(t)$ can only hold, if either the carrier velocity or its derivative give a negative contribution.

At the detection process, it becomes even clearer that the velocity term must be responsible for the reduction of the detected current $j_{\text {rec }}^{\prime}(t)$, because it scales directly with $n^{\prime}\left(P_{\text {opt }}\right)$ : 


$$
j_{r e c}^{\prime}(t) \propto e n^{\prime}(t) v^{\prime}(t)
$$

Due to the relatively fast trapping rate and slow electronhole-recombination rate, the $\mathrm{CW}$ is assumed to accumulate a considerable population in the trap states and hence slow down the trapping by trap saturation. This, in turn, would result in a carrier accumulation in the conduction band during the pulse. If $n^{\prime}(t)$ were dominant, only an amplitude increase could be expected. However, the relative number of CWgenerated conduction band carriers contributing to the $\mathrm{THz}$ current is assumed comparatively low, because of the fast trapping rate and the small ratio of optical pulse duration and pulse cycle. In contrast, when an average DC current is measured, the total number of CW-excited carriers contributes to the current, so that a growing $n^{\prime}(t)$ dominates over the shrinking velocity term.

According to the Drude-Lorentz model of carrier transport, which is widely used for modelling $\mathrm{THz}$ photo switches, because under conventional operation conditions carrier densities are high enough to restore equilibrium on a femtosecond timescale [15], $v(t)$ is expressed as the product of the elementary charge $e$, the carrier momentum relaxation time $\delta \tau$, the driving electric field and the inverse effective mass $m_{\text {eff }}{ }^{-1}: v(t)=e \cdot \delta \tau \cdot E \cdot m_{\text {eff }}{ }^{-1}$ To lower $v^{\prime}(t)$ with respect to $v(t)$, the factor $\delta \tau \cdot E \cdot m_{\text {eff }}^{-1}$ has to decrease, if a CW is added.

The most straightforward explanation is to assume a reduced local electric field through field screening occurring in $\mathrm{THz}$ photoconductive antennas [15-17]. Bias field screening occurs due to space charge fields, which attenuate the external field to make up a lower local field. Since CWexcited carriers are not supposed to accumulate in the conduction band, but populate the trap states, they are localized and hardly able to alter the screening potential. Besides, the minor number of $\mathrm{CW}$-excited carriers remaining in the conduction band or lifted into the conduction band in temporal vicinity to the pulse impact is a negligible fraction compared to the pulse-excited carriers and should not explain the observed amplitude variations. Moreover, as it has been shown, different levels of field screening lead to reshaping of the waveforms [16, 17], but the experimental results do not reveal any reshaping. Consequently, field screening does not seem to be the cause of the measured amplitude reduction. A further argument against this origin is that the receiver PCA lacks a static electric field able to separate carriers to buildup a space charge field. Thus, only the THz-field itself could create such, but since its strength is orders smaller than the emitter's bias field, it does not explain the higher impact of the $\mathrm{CW}$ in the receiver.

Observations of varying conductivity due to dynamic average effective masses have been discussed in the context of $\mathrm{THz}$ wave emission [18-22]. In those, energy-dependent excitations into satellite-valleys and intra-band relaxation have been declared responsible for these alterations. In our experiments, however, the excitation energy (photon wavelength) of the optical CW as well as of the pulsed photon energy is held constant and additional field screening by the $\mathrm{CW}$ is negligible, thus, the distribution of electrons in the conduction band states is maintained and the average $m_{e f f}$ is not subject to change.

In contrast, increasing the CW optical power leads to heating, which on a microscopic level translates to a decrease of the momentum relaxation time $\delta \tau$, because the number of scattering events is raised, when optical energy is transferred to the crystal lattice. Scattering is supposed to increase due to the excitation of LO-phonons and a higher carrier density [14, 23]. Hereby the former make the major contribution, since Monte Carlo-simulations have shown that carrier-to-carrierscattering plays only a minor role, being of elastic nature and thus only causing slight angular deviations of carrier trajectories [24]. Whereas a rise of pulsed optical power merely results in an increase of involved carriers, CW-excited carrier contribution is negligible.

Although in small bandgap materials, like InGaAs, heating by elevated bias voltages or optical fluxes can result in further heating by activation of intrinsic carriers [25, 26], this effect is not relevant in the devices reported here as indicated by the absence of runaway effects at certain power and by the fact that the reduction in amplitude is more severe for the receiver than for the emitter. If a further significant carrier activation, due to its bias the emitter should be affected more than the receiver is.

To prove the momentum relaxation time as the responsible parameter, experimental results were compared with an analysis of the response of photoconductive switches at $\mathrm{THz}$ generation and detection, provided by Duvillaret et al. [27]. Therein, the following dependences of the photocurrent in the receiver on all the time constants can be pointed out like: 


$$
\begin{aligned}
& j_{r e c}\left(t, \tau_{e m}, \tau_{r e c}, \delta \tau_{e m}, \delta \tau_{r e c}\right) \\
& \propto \frac{\delta \tau_{e m} \tau_{r e c}^{2} \delta \tau_{r e c}}{\left(\tau_{r e c}+\delta \tau_{r e c}\right)\left(\tau_{e m}+\tau_{e m}\right)\left(\frac{\tau_{e m} \delta \tau_{e m}}{\tau_{e m}+\delta \tau_{e m}}+\tau_{r e c}\right)} \\
& \cdot f\left(t, \tau_{e m}, \tau_{r e c}, \delta \tau_{e m}\right)
\end{aligned}
$$

in the time-domain and

$$
\begin{aligned}
& j_{r e c}\left(\omega, \tau_{e m}, \tau_{r e c}, \delta \tau_{e m}, \delta \tau_{r e c}\right) \\
& \propto \frac{\tau_{r e c}^{2} \delta \tau_{r e c}}{\left(\tau_{r e c}+\delta \tau_{r e c}\right)\left(1+i \omega \tau_{r e c}\right)} \\
& \cdot \frac{\tau_{e m}^{2} \delta \tau_{e m}}{\left(\tau_{e m}+\delta \tau_{e m}\right)\left(1-i \omega \tau_{e m}\right)\left(1-i \omega \frac{\tau_{e m} \delta \tau_{e m}}{\tau_{e m}+\delta \tau_{e m}}\right)}
\end{aligned}
$$

in the frequency-domain, where $\tau_{e m}$ and $\tau_{r e c}$ denote the electron lifetimes, $\delta \tau_{e m}$ and $\delta \tau_{r e c}$ the momentum relaxation times of emitter and receiver, respectively. In the first expression the term denoted $f\left(t, \tau_{e m}, \tau_{r e c}, \delta \tau_{e m}\right)$ abbreviates a group of exponential and inverse error functions, in which only $\tau_{e m}, \tau_{r e c}$ and $\delta \tau_{e m}$, but not $\delta \tau_{r e c}$ appear as arguments. Above equations were used to compute temporal pulse forms and corresponding Fourier spectra under variation of carrier lifetimes and momentum relaxation times. In the regime of carrier lifetimes typical for LT-InGaAs/InAlAs ( $\sim 0.7 \mathrm{ps}$ ), an increase in carrier lifetime results in reshaping the pulse form as can be seen in Figs. 6a and 6b. Whereas in the receiver, for increasing carrier lifetimes, the leading positive peak is lowered and its energy transferred increasingly to the negative peak, in the emitter, exactly the opposite takes place; the positive peak grows, while the negative one reduces. Measured signals, however, show a uniform decrease in the positive as well as the negative peak (see Fig. 3), as opposed to the energy redistribution between both peaks observed in Figs. 6a and 6b. Furthermore, the temporal pulse widths broaden and the spectral peaks consequently shift, as it is depicted in Figs. 8a and 8b. Carrier lifetime variations, which cause amplitude changes in the order of the measured ones ( $\sim 35 \%$ for the receiver, see Fig. 2 ), result in shifts of the maximum frequency of the order of $\Delta f\left(P S D_{\max }\right)=$ $100 \mathrm{GHz}$. Since within the resolution limit of the measurements $(\Delta f=20 \mathrm{GHz})$ no noticeable spectral shift is observed (see Fig. 4), the effect should not be attributed to a change of the carrier lifetime. The situation becomes different, when the momentum relaxation times are varied. For smaller $\delta \tau_{e m}$ or $\delta \tau_{r e c}$ the amplitude becomes smaller in both peaks (Figs. 6c and $6 \mathrm{~d}$ ), while neither pulse shape nor spectrum do significantly change, just as observed in the measurements. Computed spectra, however, reveal a difference in the behavior of the emitter and receiver PCA. A variation of $\delta \tau_{e m}$ causes a divergence at higher frequencies (Fig. 7d). A variation of $\delta \tau_{\text {rec }}$, in contrast, leaves the Fourier spectrum unchanged (Fig. 7c). This becomes clear considering Eq. 6: Whereas $\delta \tau_{e m}$ affects the pulse shape (being argument of term $f\left(t, \tau_{e m}, \tau_{r e c}, \delta \tau_{e m}\right)$ ), and, in turn, affects the spectral distribution, $\delta \tau_{\text {rec }}$ plays a different role: Because it does not appear in $f\left(t, \tau_{e m}, \tau_{r e c}, \delta \tau_{e m}\right)$, but in the fractional term, there is no impact on the pulse shape, but a reciprocal scaling in amplitude, which corresponds to the one measured in Fig. 5. Hence, the spectral power distribution obtained under variation of $\delta \tau_{r e c}$ is maintained. The slight differences in the emitter spectra, when the CW is injected, cannot be appreciated in the experiments due to bandwidth and noise limitations in the setup.

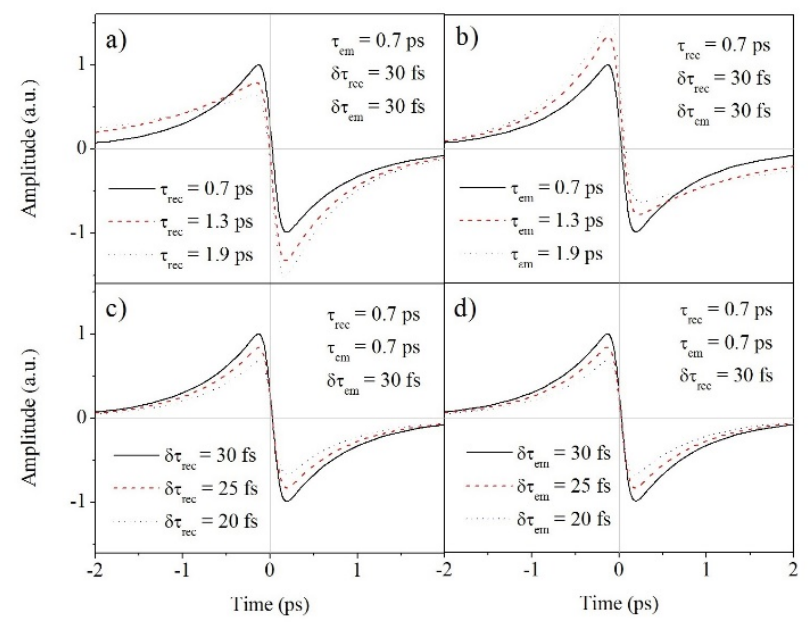

FIG. 6 Computed time traces of receiver photocurrents under variation of carrier lifetimes (a, b) and momentum relaxation times (c, d) of the receiver (a, c) and the emitter (b, d)

To back up the conclusions drawn above, a photocurrent autocorrelation measurement was conducted for the emitter module. Autocorrelation traces allow the extraction of the lifetime of the conduction carriers [28]. As it can be seen in Fig. 8, the irradiance of an optical CW of similar power of the pulsed power, which noticeably reduces the radiated $\mathrm{THz}$ field amplitude, does not recognizably alter the autocorrelation trace within the limits of the resolution of the measurement. Gaussian fits of the principal lobe show almost identical FWHMs of around 230 fs. The slightly smaller width of the graph representing the additional CW cannot be taken as an indicator for a narrowing due to the measurement uncertainty. A potential increase of the carrier lifetime, however, would favor higher $\mathrm{THz}$ amplitudes (compare Fig. 7b), what is in opposition to the observed behavior. 


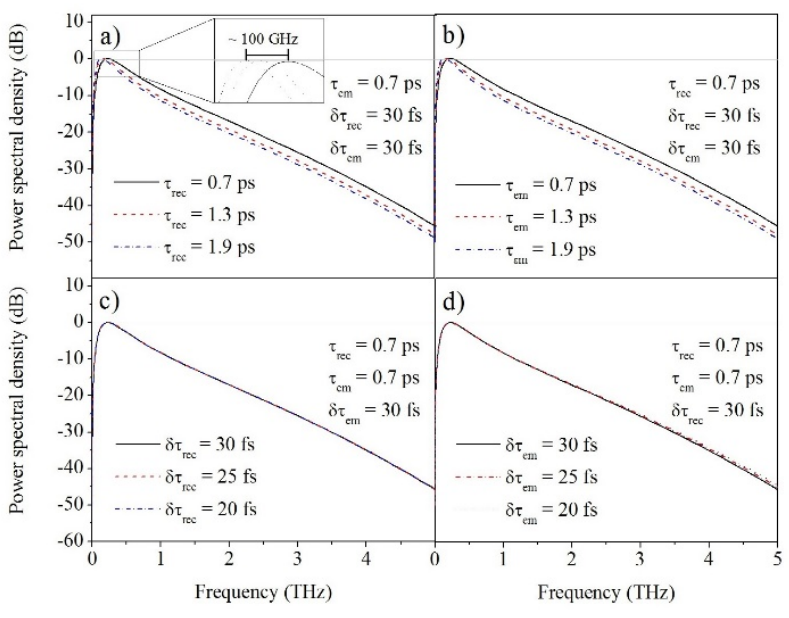

FIG. 7 Computed spectra of receiver photo-currents under variation of carrier lifetimes (a, b) and momentum relaxation times (c, d) of the receiver (a, c) and the emitter (b, d)

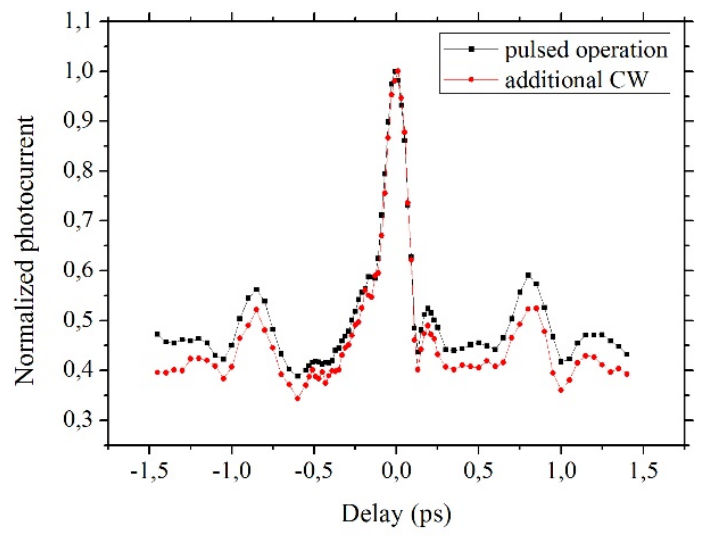

FIG. 8 Photocurrent autocorrelation trace for the emitter module.

\section{CONCLUSION}

In this work, the injection of an additional continuous wave optical signal into both emitter and receiver antennas in a THz time-domain spectrometer has been investigated. In contrast to previous observations made with a GaAs emitter chip excited at $800 \mathrm{~nm}$ [29], CW optical power diminishes the amplitude of the $\mathrm{THz}$ field, while the signal bandwidth was maintained. CW injection into the receiver chip leads to an amplitude reduction as well, but with a higher efficiency. This counterintuitive behavior of increasing the optical flux and hence the number of carriers while reducing the $\mathrm{THz}$ field amplitude can be understood considering the variations of the carrier momentum relaxation time in the photoconducting switch implicated by the CW. Computations suggest that the amplitude reduction does not result from the variation of the free carrier density and thus carrier lifetime extension by trap saturation, but is caused by the reduced momentum relaxation time, which makes the rising photocurrent see a higher resistivity at the moment of the pulse arrival. That means that the energy transfer responsible for this effect takes place via phonon creation by scattering processes, i.e. heat generation, rather than electronic transitions by photon absorption.

In spite of its potential to implement an all-optical modulation method for $\mathrm{THz}$ signals [30], this effect might degrade the performance of $\mathrm{THz}$ sensing systems with remote, distributed feeding using optical amplification [6], since the residual amplified spontaneous emission (ASE) noise results in a continuous component. However, in standard systems with a single amplification stage, ASE power is small and the associated degradation can be neglected.

\section{ACKNOWLEDGMENTS}

The authors gratefully acknowledge Dr. Lionel Duvillaret for help with his analytical model. This work was partially supported by the Spanish Ministry of Economy and Competitiveness through project TEC2012-35797 and FPU grant $12 / 02847$.

\section{REFERENCES}

[1] P.-U. Jepsen, D. G. Cooke and M. Koch, "Terahertz spectroscopy and Imaging - Modern techniques and applications,” Laser Photon. Rev.,” vol. 5, no. 1, pp. 124 166, Jan. 2011.

[2] M. Tonouchi, "Cutting-edge terahertz technology," Nature Photon., vol. 1, no. 97, Feb. 2007.

[3] P. H. Siegel, "Terahertz Technology," IEEE Trans. Microw. Theory Tech., vol. 50, no. 3, pp. 910 - 928, Mar. 2002.

[4] B. Sartorius et al., "All-fiber terahertz time-domain spectrometer operating at $1.5 \mu \mathrm{m}$ telecom wavelengths," Opt. Expr., vol. 16, no. 13, pp. 9565 - 9570, Jun 2008.

[5] B. Sartorius et al., "Continuous wave terahertz systems exploiting $1.5 \mu \mathrm{m}$ telecom technologies,” Opt. Expr., vol 17, no. 17, pp. 15001 - 15007, Aug. 2009.

[6] A. Bockelt, J. Palací, and B. Vidal, "All-fiber Centralized Architecture for Parallel Terahertz Sensors,” IEEE Trans. Terahertz Sci. Technol., vol. 5, no. 1, pp. 137 - 144, Jan. 2015.

[7] R. J. B. Dietz et al., "THz generation at $1.55 \mu \mathrm{m}$ excitation: six-fold increase in $\mathrm{THz}$ conversion efficiency by 
separated photoconductive and trapping regions,” Opt. Expr., vol. 19, no. 27, pp. 25911 - 25917, Dec. 2011.

[8] R. J. B. Dietz et al., "64 $\mu \mathrm{W}$ pulsed terahertz emission from growth optimized InGaAs/InAlAs heterostructures with separated photoconductive and trapping regions," Appl. Phys. Lett., vol. 103, Aug. 2013.

[9] H. Roehle et al., "Next generation $1.5 \mu \mathrm{m}$ terahertz antennas: mesa-structuring of InGaAs/InAlAs photoconductive layers," Opt. Expr., vol. 118, no. 3, pp. 2296 - 2301, Feb. 2010.

[10] S. C. Corzo-García, M. Alfaro and E. Castro-Camus, "Transit time enhanced bandwidth in nanostructured terahertz emitters,” J. Infrared Milli Terahz Waves, vol. 35, no. 12, pp. 987 - 992, Dec. 2014.

[11] A. Krotkus, "Semiconductors for terahertz photonics applications,” J. Phys. D: Appl. Phys., vol. 43, no. 27, 273001 (21 pp), Jun. 2010.

[12] Y.-S. Lee, "Principles of Terahertz Science and Technology,” Springer, New York, 2009.

[13] B. Globisch et al., "Carrier dynamics in Beryllium doped low-temperature-grown InGaAs/InAlAs,” Appl. Phys. Lett., vol. 104, no. 17, 172103 (pp. 4), Apr. 2014.

[14] R. Yano et al., "Systematic pump-probe terahertz wave emission spectroscopy of a photoconductive antenna fabricated on low-temperature-grown GaAs,” J. of Appl. Phys., vol. 96, no. 7, pp. 3635 - 3638, Oct. 2004.

[15] P. Uhd Jepsen, R.H. Jacobsen and S.R. Keiding, "Generation and detection of terahertz pulses from biased semiconductor antennas,” J. Opt. Soc. Am. B, vol. 13, no. 11, pp. 2424 - 2436, Nov. 1996.

[16] J.E. Pedersen et al., "Ultrafast local field dynamics in photoconductive THz antennas,” Appl. Phys. Lett., vol. 62, no. 11, pp. 1265 - 1267, Dec. 1992.

[17] G. Rodríguez and A.J. Taylor, "Screening of the bias field in terahertz generation from photoconductors," Opt. Lett., vol. 21, no. 14, pp. 1046 - 1048, Jul. 1996.

[18] A.J. Taylor, P.K. Benicewicz and S.M. Young, "Modeling of femtosecond electromagnetic pulses from large-aperture photoconductors,” Opt. Lett., vol. 18, no. 16, pp. 1340 - 1342, Aug. 1993.

[19] P.K. Benicewicz and A.J. Taylor, "Scaling of terahertz radiation from large-aperture biased InP photoconductors," Opt. Lett., vol. 18, no. 16, pp. 1332 - 1334, Aug. 1993.
[20] G. Rodríguez, S.R. Cáceres and A.J. Taylor, “Modeling of terahertz radiation from biased photoconductors: transient velocity effects,” Opt. Lett., vol. 19, no. 23, pp. 1994 - 1996, Dec. 1994.

[21] J.-H. Son, T.B. Norris and J.F. Whitaker, "Terahertz electromagnetic pulses as probes for transient velocity overshoot in GaAs and Si," J. Opt. Soc. Am. B, vol. 11, no. 12, pp. 2519 - 2527, Dec. 1994.

[22] C. Ludwig and J. Kuhl, "Studies of the temporal and spectral shape of terahertz pulses generated from photoconducting switches,” Appl. Phys. Lett., vol. 69, no. 9, pp. 1194 - 1196, Jun. 1996.

[23] E. Castro-Camus, J. Lloyd-Hughes and M.B. Johnston, "Three-dimensional carrier dynamics simulation of terahertz emission from photoconductive switches,” Phys. Rev. B, vol. 71, no. 19, 195301 (pp. 7), May 2005.

[24] E. Castro-Camus, M. B. Johnston and J. Lloyd-Hughes, "Simulation of fluence-dependent photocurrent in terahertz photoconductive receivers," Semicond. Sci. and Technol., vol. 27, no. 11, 115011 (pp. 6), Sept. 2012.

[25] S. Preu et al, “1550 nm ErAs:In(Al)GaAs large area photoconductive emitters,” Appl. Phys. Lett., vol. 101, no. 10, 101105 (pp. 4), Sept. 2012.

[26] M. Mittendorff et al, "Large area photoconductive terahertz emitter for $1.55 \mu \mathrm{m}$ excitation based on an InGaAs heterostructure,” Nanotechnology, vol. 24, no. 21, 214007 (pp. 7), Apr. 2013.

[27] L. Duvillaret, F. Garet, J.-F. Roux and J.-L. Coutaz, Analytical modeling and optimization of terahertz timedomain spectroscopy experiments using photoswitches as antennas," IEEE J. on Selected Topics in Quant. Electr., vol. 7, no. 4, pp. 615 - 623, Jul. 2001.

[28] T. F. Carruthers and J. F. Weller, "Picosecond optical mixing in fast photodetectors,” Appl. Phys. Lett., vol. 48, no. 7, pp. 460 - 462, Feb. 1986

[29] C. Ryu and S.G. Kong, "Boosting terahertz radiation in THz-TDS using continuous-wave laser,” Electr. Lett., vol. 46, no. 5, pp. 359 - 360, Mar 2010.

[30] A. Bockelt, J. Palací and B. Vidal, “Control of terahertz emission in photoconductive antennas through an additional optical continuous wave,” Opt. Lett., vol. 38, no. 16, pp. 3123 - 3125, Aug. 2013. 